\section{Making science a good read}

SIR - The leading article "Must science be impenetrable?'" (Nature 305, 477; 1983) concluded that little or nothing could be done to improve the situation, and that the authors are the most obvious culprits. I argue that the poor quality of illustrations contributes towards impenetrability, and that on this point the editors are the culprits.

The decipherment of a figure that contains more than one part can be very laborious, because the pertinent information is often buried in an overcrowded legend. This could be avoided if the following rules were applied:

(1) When the graph includes more than one line, identify each by appropriate labelling. (2) When the figure contains several panels with the same abscissa and the same ordinate, usually identified as $(a),(b)$ and so on, indicate on each panel its experimental specificity.

(3) Whenever possible, use the same symbol for the same experimental condition in all comparable figures throughout the article.

(4) When several symbols need to be used in a graph, use the open circle for the control condition; for instance, if an experiment has been performed without (o) and with ( $\bullet$ ) an addition or a treatment, use the symbols as indicated. (The open

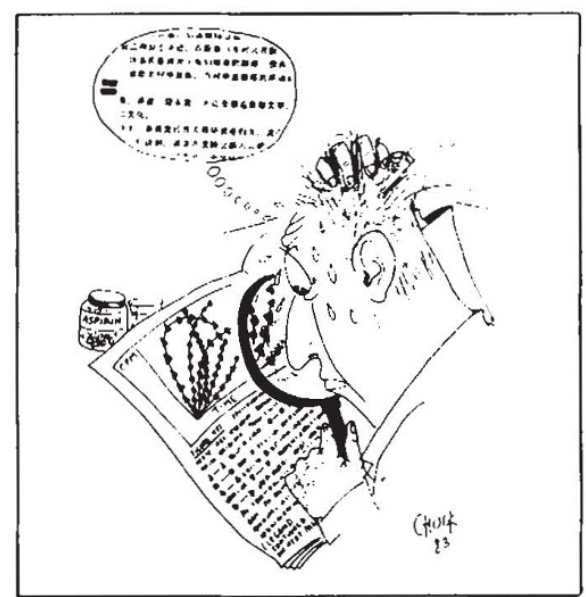

circle is the simplest symbol available, and its "emptiness" suggests that nothing has been added.) Rule (4) should prevail over rule (3) when these two rules are in conflict.

(5) Make the labelling of abscissa and ordinate as precise as possible. For instance, it is preferable to write "Time after drug addition" than simply "Time". Also express results in moles rather than in c.p.m. or in absorbency units, sparing the reader the task of doing the conversion. If results are given in per cent, give the value of 100 per cent in standard units in the legend.

(6) The legend should be as short as possible, and could sometimes be restricted to the title since, ideally, the figure would be fully intelligible without making reference to the legend.

From my own experience of the biochemical literature, I know that, if labelling of figures is still very limited, it is because it is systematically discouraged by subeditors, whose policy is to push all the information into the legends. With modern technology, the cost of lettering can no longer be an excuse for this negative attitude.

\section{Université Catholique de Louvain and International Institute of Cellular and Molecular Pathology, UCL 75.39, Avenue Hippocrate 75, B-1200 Brussels, Belgium}

\section{Nicotine delivery}

SIR - The concern of the British Royal College of Physicians (Nature 1 December 1983, p.418) with smoking and health is justified and well directed towards the immediate problem of reducing the number of people who may be influenced by cigarette advertising. The problem though, is that cigarette smoking is a means of drug delivery that is both crude and effective. If one assumes that some number of people in any society want to use drugs for a variety of reasons and that smoking is merely a way of delivering an addictive drug, the problem becomes conceptually simpler.

Addiction to the natural alkaloid nicotine is widespread and social tolerance of the drug and its users is such that for years a debate existed over whether chronic users were actually addicted in the same sense as opiate users. Semantic differences apart, smokers mostly smoke to deliver nicotine to their mucus membranes so it can be absorbed into the blood.

Alternative routes of drug administration more cosmetic than chewing tobacco or snuffs should be developed so that the nicotine addict has alternatives to cigarettes. Nicotine chewing gum has had limited success, but may soon become available worldwide. Another alternative might be transdermal application much in the manner of nitroglycerine and scopolomine patches. Nicotine "inhalers" might also be feasible if dosage could be adjusted.

I would appreciate receiving correspondence from individuals or groups actively engaged in finding alternative methods of nicotine delivery in humans.

CECII. H. FOX

8708 First Avenue,

Silver Spring, Maryland 20910, USA

\section{Prudent antibiotics}

SIR - A leading article in Nature in 1981 (Nature 292, 661) referred to the publication of the "Statement Regarding Worldwide Antibiotic Misuse", which was signed by more than 200 physicians and scientists and intended as an alert to the problem of antibiotic abuse.

The article raised the question of how the problem would continue to be addressed. The response generated by the statement enabled the Alliance for the Prudent Use of Antibiotics (APUA) to be established at the end of 1981. Its members include physicians, scientists, medical and public health personnel and members of the general public from more than 50 countries. The alliance has adopted a positive rather than a negative approach by advocating and defining good usage. Its activities include the gathering and dissemination of information and education on antibiotics and their use. The alliance has begun to organize a network of groups and individuals who are working in their own countries to improve antibiotic usage. The APUA Newsletter has opened a line of communication among the members. We recognize that it is at the level of the consumer - patient, physician, farmer, gardener, pet owner, beekeeper - that the rational use of antibiotics must be understood and accepted.

It is our long-range objective to implement changes in the use of antibiotics which will preserve their therapeutic effectiveness.

Alliance for the Prudent Use

Stuart B. LeVY of Antibiotics,

PO Box 1372,

Boston, Massuchussetts 02117, USA

\section{Gripes of wrath}

SIR - I read Walter Gratzer's review of Fowler's Modern English Usage (Nature 10 November 1983, p.134) and was both saddened and amused at the examples he quoted of uncouth English.

My own pet irritation is the use of "in the order of" in place of "of the order of". This has become very common, but I do not recall ever coming across it more than twenty years or so ago. I believe it is an example of a phrase that "escaped" from scientific usage, was mangled by those who do not understand the meaning of order of magnitude, and then was hypnotically readopted by all because of the frequency of its appearance in the distorted form.

Other pet aversions of mine include "anticipate" when "expect" is meant (we anticipate completion by the end of the year), and the introduction of a quite inappropriate conditional qualification (symposium programmes are available in the foyer if delegates care to help themselves).

Finally, an ambiguity in a classified advertisement seen many years ago which never fails to amuse me when I recall it: Miss E. Brown, wardrobe dealer, having cast off clothing of all descriptions, invites inspection.

DENNIS PIPER

Abingerwood, Ewhurst, Cranleigh, Surrey GU6 7SJ, UK 